\title{
A Mobile Application Flow Representation for Mutual Understanding of IT and Healthcare Professionals
}

\author{
Yusuf Nasuh Erturan ${ }^{1}$, Semih Bilgen ${ }^{2}$, Gul Tokdemir ${ }^{3}$, Nergiz E. Cagiltay ${ }^{4}$, \\ Ekrem Yildiz ${ }^{5}$, and Esra Özcebe ${ }^{6}$ \\ ${ }^{1}$ Department of Medical Informatics - METU, Ankara, Turkey \\ nasuherturan@gmail.com \\ ${ }^{2}$ Electrical Engineering Department - METU, Ankara, Turkey \\ bilgen@metu.edu.tr \\ ${ }^{3}$ Department of Computer Engineering, Cankaya University, Ankara, Turkey \\ gtokdemir@cankaya.edu.tr \\ ${ }^{4}$ Department of Software Engineering, Atılım University, Ankara, Turkey \\ nergizatilim.edu.tr \\ ${ }^{5}$ Department of Information Sciences, METU, Ankara, Turkey \\ ekrem.yildiz@live.com \\ ${ }^{6}$ Faculty of Education, International Cyprus University, Lefkosa, Cyprus \\ eozcebe@gmail.com
}

\begin{abstract}
Ever since mobile applications were developed and became popular, they have started to take part in almost every field of our lives. Healthcare is one of the most popular fields that mobile applications have become a part of. However, development of mobile healthcare applications requires an interdisciplinary work on which people from different domains should communicate. To do so efficiently, mobile application instructions should be provided as clearly as possible so that mutual understanding can be achieved. This study, aims to provide a methodology to provide the common grounds for healthcare and IT specialists so that to improve the satisfaction level of all the stakeholders of the system from the provided IT services and the end-user interfaces. In other words, by providing a better communication medium for the stakeholders during the design phase, we believe that software development process will be improved, so do their satisfaction from the developed system.
\end{abstract}

Keywords: Mobile healthcare, Communication gap, Representation guideline.

\section{Introduction}

Ever since the mobile applications were developed and became popular, they have started to take part in almost every field of our lives. Healthcare is one of the most popular fields that mobile applications have become a part of. According to a report written by Heather Clancy and published by Mobile Healthcare in 2011, over the next four years, mobile healthcare applications will change the way doctors communicate with each other, their work operations and also the way how healthcare organizations 
interact with patient communities (Clancy, 2011). The vision of Clancy has already started to come true. A study which was conducted by "Pyramid Research Group" (HealthcareITNews, 2010) in 2011 showed that, more than 200 million mobile health applications were in use by doctors and patients and this number would be tripled in 2012. Another result from that study is that, 70 percent of people worldwide are interested in having access to at least one m-health application and they are willing to pay for it (HealthcareITNews, 2010). Furthermore, mobile healthcare application market was $\$ 718$ million in 2011 and it would reach $\$ 1.3$ billion in 2012 (Research2guidance, 2012). Another report which was prepared by Arthur D. Little Co. in 2011 also stated that mobile health potential value will be $\$ 10$ billion within the next five years (Arthur, 2011).This is a great appetizer for IT companies, so they develop new strategies to add mobile healthcare into their future plans.

Healthcare IT is an interdisciplinary field and it is much more complicated than other interdisciplinary fields, because health issues are critical in terms of patients' lives. Moreover, integration of mobile technologies into healthcare (especially into mobile healthcare applications) is one of the hot topics in the field. It is because the market share of mobile health applications is so huge, that the numbers are expressed in billions of dollars. In such a profitable market, IT companies have started to invest in mobile healthcare to get a market share.

'Mobile healthcare IT applications'; just the phrase itself includes different domains: Mobile, healthcare, IT and application domain. As it can be inferred, developing a mobile healthcare IT application requires gathering of people from different expertise. Gathering those people is the easy task; however, making them work on the same subject and communicate through the same language may not be that easy. Uniting all those people in a team requires utmost commitment to overcome the communication gap, since the application developers do not have the related medical knowledge and the medical staff don't understand the software code unless they are provided a human-readable visualization (Ongenae, 2010). To overcome this problem, a document, which facilitates mutual understanding, should be provided to all those people. There are different kinds of guidelines developed for this purpose. However, if the guidelines are in a mass detailed format, the size of the guidelines may become a problem and if they are superficially prepared, they can be vague to interpret (Backere, Steurbaut, Colpaert \& Decruyenaere, 2010).

People from different domains - in our case IT people and healthcare specialists usually have different ways of thinking. Studies show that people from healthcare domain and technical domain are from different cultures, they use different languages to communicate, they do not know each other's domains and because of this reason the technology integration in medical domain requires these people to come together and work closely (Dankelman, Grimbergen \& Stassen, 2007). However people from healthcare domain are usually too busy to spend time on such collaborations and it is not always possible these two groups of people come together and work together effectively. Kilov and Sack (2007) state that communication of experts from different domains is only possible through a joint ontology and creation of this ontology 
requires a common system of concepts which are applicable and extensible to any specific viewpoint (Kilov \& Sack, 2009). At this point the question is how these common concepts will be created and what these common concepts will be.

In this study, we aim to provide a methodology to provide the common grounds for healthcare and IT specialists. The main idea behind this developed methodology is to improve the satisfaction level of all stakeholders of the system from the provided IT services and the end-user interfaces. In other words, by providing a better communication medium for the stakeholders during the design phase, we believe that software development process will be improved, so do their satisfaction from the developed system.

Specifically in this study, the mobile application development process is analyzed with this aim. We provide a clear and efficient MAFR that can be used by IT professionals as well as healthcare professionals during mobile healthcare application development process, to ensure mutual understanding of the functionality and the interface specifications of the mobile application. In order to illustrate the application of our proposal, a mobile healthcare application developed specifically for speech and language therapy of children with Speech Sound Disorder has been examined. The effectiveness and usability of the proposed MAFR is tested during the design of a mobile health application, which involves four domain experts. In this study, the development stages of the system are well documented to better understand the communication problems of these specialists and how the proposed approach addresses these problems. Additionally, an observer observed all communication stages during the application of the proposed methodology and interviews with stakeholders are conducted to better understand the effectiveness of the proposed representation. The study analyses the collected data to explore the effect of proposed approach to improve the communication between these groups of people and to increase the success of the project in terms of development time and effectiveness.

The data collected from this study is analyzed descriptively to better understand the effect of the proposed communication tool on the quality and success of the developed software system.

\section{Mobile Application Flow Representation (MAFR)}

Mobile Application Flow Representation (MAFR) includes representation elements, explanation of these elements, and the MAFR methodology that is used during mobile application design phase.

\subsection{Representation Elements}

Representation elements are the shapes that are shown to the users about the mobile application interaction design. These elements consist of gestures, pages, buttons, pictures, videos, text fields as presented in Table 1. 
Table 1. Representation Elements

\begin{tabular}{|c|c|c|}
\hline Notation & Name & Explanation \\
\hline & Touch & Touch on the related element. \\
\hline & $\begin{array}{l}\text { Touch and } \\
\text { Hold }\end{array}$ & $\begin{array}{l}\text { Touch on related element and hold your } \\
\text { finger for a while }\end{array}$ \\
\hline & $\begin{array}{l}\text { Slide right or } \\
\text { Slide left }\end{array}$ & $\begin{array}{l}\text { Touch on the element and with the finger } \\
\text { touched on it; slide it to the right or to the } \\
\text { left. }\end{array}$ \\
\hline & Zoom in & $\begin{array}{l}\text { Touch on the related element with two } \\
\text { fingers and with the fingers touched on it, } \\
\text { zoom in the related element. }\end{array}$ \\
\hline & Zoom out & $\begin{array}{l}\text { Touch on the related element with two } \\
\text { fingers and with the fingers touched on it, } \\
\text { zoom out the related element. }\end{array}$ \\
\hline & Drag object & $\begin{array}{l}\text { Touch on the related element and with the } \\
\text { finger touched on it, drag the related ele- } \\
\text { ment. }\end{array}$ \\
\hline & Action lines & $\begin{array}{l}\text { Action line direct the user to the related } \\
\text { page based on the users actions. This line } \\
\text { shows that what the application will display } \\
\text { next. }\end{array}$ \\
\hline & $\begin{array}{l}\text { Explanation for } \\
\text { the action line }\end{array}$ & $\begin{array}{l}\text { Used for explaining the action line. For } \\
\text { example, if the related item is a touchable } \\
\text { item, the explanation will be }<\text { touched }>\text {. }\end{array}$ \\
\hline & $\begin{array}{l}\text { Touchable } \\
\text { button }\end{array}$ & Shows that user can touch on the button. \\
\hline & $\begin{array}{l}\text { Touchable } \\
\text { combobox } \\
\text { button }\end{array}$ & $\begin{array}{l}\text { Shows that user can touch on the combo- } \\
\text { box therefore, the list in the combobox will } \\
\text { be showed to the user. }\end{array}$ \\
\hline & $\begin{array}{l}\text { Touchable } \\
\text { radio button }\end{array}$ & $\begin{array}{l}\text { Shows that user can touch on the items } \\
\text { listed, i.e. user will mark the item by touch- } \\
\text { ing on it. }\end{array}$ \\
\hline & $\begin{array}{l}\text { Touchable } \\
\text { checkbox } \\
\text { button }\end{array}$ & $\begin{array}{l}\text { Shows that user can touch on the items } \\
\text { listed, i.e. user will mark the item by touch- } \\
\text { ing on it. }\end{array}$ \\
\hline & $\begin{array}{l}\text { Touchable } \\
\text { input text } \\
\text { button }\end{array}$ & $\begin{array}{l}\text { Shows that user can touch on the textbox } \\
\text { and make an input. }\end{array}$ \\
\hline
\end{tabular}


Table 1. (continued)

\begin{tabular}{|l|l|l|l|}
\hline & Text Field & $\begin{array}{l}\text { Shows the content as simple text format so } \\
\text { that user can read what is written in it. For } \\
\text { example, the content of a web page. }\end{array}$ \\
\hline & $\begin{array}{l}\text { Touchable } \\
\text { Image }\end{array}$ & Shows that user can touch on the image. \\
\hline & $\begin{array}{l}\text { Touchable } \\
\text { Video }\end{array}$ & Shows that user can touch on the video. \\
\hline
\end{tabular}

For clarification, explanations should be as detailed as possible. For instance, if the application has a main page with 5 touchable buttons on it, they are pictured and coded as follows:

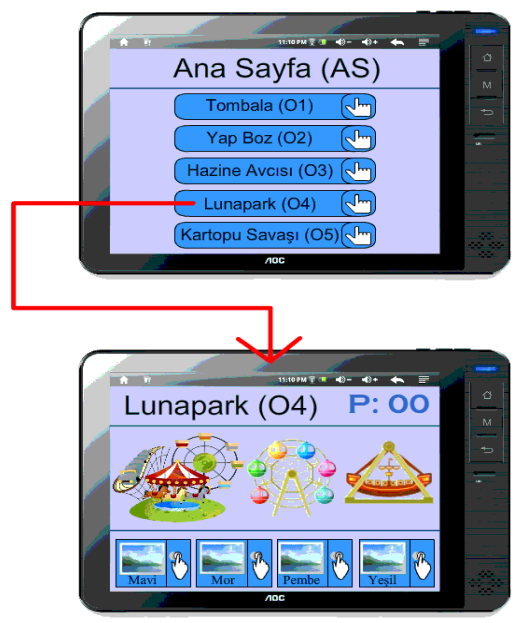

Fig. 1. Example Notation

This example shows that there is a main page including 5 touchable buttons on it. When a user touches a button, the flow goes to the related page. In this example when the user touches on 4th touchable button the flow goes to "Lunapark (O4)" game page.

Action line directs the user to the related page based on the user's actions. This line shows that what the application will display next. In figure 1, there are two interfaces. First one is the main page and the other one is the page activated based on the user's action. Transitions are showed with the arrows. Second page, which is named as "Lunapark (O4)", has 3 images, 4 drag objects and the score. 


\section{Case Study}

The proposed MAFR approach is applied in development of a mobile application for speech and language disorder domain. The case study aims to test the proposed MAFR that aims to eliminate the communication gap between therapist and mobile software developers especially for a speech and language disorder application. Speech and Language disordered patients refers to a group of people who have deficits in both speech (fluency, rate, or articulation) and language (comprehension, expression, or usage) (Dennis \& Baker, 2002). Patients who have speech and language disorder need to perform some practices which are guided by therapists to eliminate the disorder effects. In order to carry out those practices patients should meet with their therapist very often. However, some patients especially who live in rural areas do not have an opportunity to meet with therapists frequently. Speech and language disorder mobile application helps to solve this problem for the patients and therapists. The mobile application for this purpose should be developed same as what the subject matter exactly does in practices. Therefore, the application should reflect the real practice as much as possible, which requires broad communication of therapist and application developers. For the case study, a mobile application design is performed using the proposed MAFR with the aim of providing a common communication medium between therapist and developers.

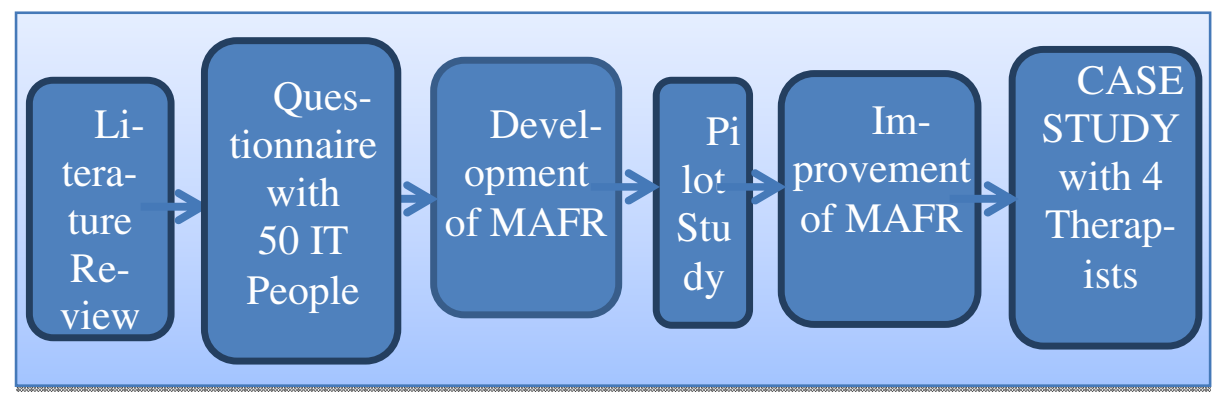

Fig. 2. Research Flow

As shown in Figure 2, first of all, a literature study on mobile application design guidelines was performed. After the analysis of literature review, a questionnaire was conducted to 50 people from IT domain and gathered their experiences about the problems that they had faced with people from other domain during development process. Then, MAFR was developed and a pilot study with one therapist was performed to evaluate MAFR and the methods that are applied to the patients during the therapy. According to the results of the pilot study, MAFR was improved and then a case study with four therapists was conducted and after the interviews therapists' views about MAFR were collected. One of the therapists has 20 years of experience in language and speech disorder area, who was also a child development and education specialist. The second therapist has 12 years of experience in language and speech disorder area who was a hearing-impaired teacher. The third therapist has 9 years experiences in language and speech disorder area. The fourth therapist has 
3 years experiences in language and speech disorder area and she had four year experience in teaching of mentally disordered people.

\subsection{MAFR of the Pilot Application}

MAFR of the pilot application which was used during the interviews with therapists is given below. These representations were shown to the therapists and their opinions were collected.

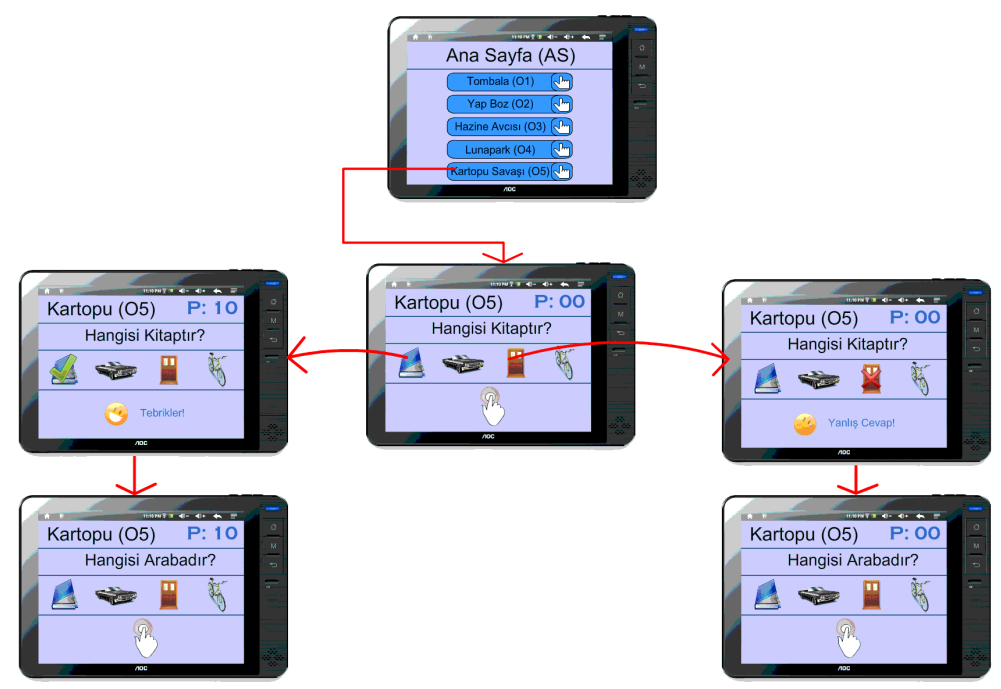

Fig. 3. MAFR of "Kartopu" Mobile Game Application

The MAFR which is shown in Figure 2 represents the "Kartopu" game. It has 6 different interfaces player interacts with including MainPage (AnaSayfa). "Kartopu" game is aimed to improve patients' vocabulary. Moreover, as they play the game their perception will also be improved since they will learn more objects. In the game, questions like "Which one is a book?", and four pictures are shown to the patients. When patient drag the snowball onto the correct object, the application will give a feedback as "Congratulations" and the patient gets 10 points, and then the new question comes to the screen. If the patient drags the snowball to the wrong object the application will give a feedback as "Wrong answer" and the patient does not get any point, and then the new question follows. At the end of the game, score and logs will be recorded to see the improvement of the patient.

\section{$4 \quad$ Results}

According to the questionnaire conducted to 50 people from IT domain, they are facing with different kinds of problems during development process. Results of the 
questionnaire showed that $50 \%$ of the attendees need new tools and models to describe the process to the customers easily. Moreover, $70 \%$ of the attendees said that those kinds of tools and models can decrease both development process of the application and cost of the software. Furthermore, $45 \%$ of the attendees mentioned that customers should be in the software development process. Besides, $71 \%$ of the attendees stated that the reason why the software projects last more than the project time and exceeds the project cost is requirements not clearly identified by the customers. $80 \%$ of the attendees stated the problem between them and customers. They said that they have communication problem because of the fact that they either can't express themselves to customers or customers can't express what they what to them. $60 \%$ of the attendees think that there is a communication gap between customers from different domains and people from IT domain. Also in open-ended questions of the questionnaire, most of the attendees mentioned about the importance of software requirements as the problems between customers and software developers are caused by unclear requirements and having difficulty in communication. Moreover, as a model used in development process, most of the attendees use pictures, slides and storyboard. So, they need a simple model that can learly understandable by both customers and people from IT domain.

From the questionnaire, it can be concluded that there is a communication gap between customers and people from IT domain when they tried to identify the requirements of the software application which will cause the exceeding of project cost and time. In order to full this gap, it would be better to have new tool or model which will not only decrease the project time and cost but also increase the effective software application since the requirements are clearly identified.

As to interviews, the interviews with four subject matter experts have lasted $30 \mathrm{mi}-$ nutes each. The questions used during the interviews are provided in Appendix A. The proposed mobile application design has included a brief explanation of the application and its design in MAFR. Each interview is recorded and transcribed for analysis. Four interviewees were asked to examine MAFR document in 10 minutes separately. After then, the interview questions were asked to the therapists to get their ideas about MAFR.

The results showed that the proposed MAFR is considered to be very important during such kind of development process in terms of effectiveness, and efficiency of the process.

A 12-year-experienced interviewee said that "using such kind of a model during development process is very effective". A 9-year-experienced interviewee told that "conversations are not always a good way and can be understood differently from person to person".

All of the interviewees stated that they are fully satisfied with this development process and using this model makes the process efficient. During the usual development process, 20-year-experienced interviewee stated that it is pointless to get subject matter experts' ideas after the development is over. She mentioned that the efficient way is correcting misunderstandings during the process. For instance, in our case all the four therapists corrected different parts of our misunderstandings and neither therapists nor software experts have to do the development twice. 
Finally, two of the interviewees stated that representation elements of this MAFR are so easy to understand that they don't even need to look at the explanations. They said that most people can easily understand the elements.

"Have you ever been in a development process of a software or application?" was one of the questions directed to interviewees. None of the therapists have been in a development process of a software or application. They just face with the application after it is developed. They stated that most of the applications they have faced with have serious problems which have to be taken into consideration in terms of relevance to subject, relevance to exercises used in the field and need of people in the target group.

"What do you think about the MAFR?", "Did you understand the representation easily?" were other questions. Therapists said that the representation elements of the MAFR are so easy to understand that they didn't even need to read the explanations.

"What are the positive and negative parts of the MAFR?" All of the interviewees stated that subject matter experts should definitely be included in the development process. However, most of the times they can't tell precisely what they want to tell the IT professionals and most of the times they don't understand what the IT professionals tell them. The positive part of this MAFR is that it is a common conversation platform for different professions. When it comes to the negative part of the MAFR, two of the interviewees stated that it shouldn't be like a storyboard and there is no need to represent all pages of the application with the MAFR. It should be as simple as it can be.

\section{Conclusion}

During the development process, IT companies have serious communication problems while facing with customers. These problems are because of either they can't efficiently express themselves to customers or customers can't tell what they really want from them. To overcome this problem they mostly use UML representations but they are not very well understood by customers, in our case healthcare professionals. Healthcare professionals on the other hand try to tell what they want verbally but since they are in a specific field and people from IT field don't understand what they want to say to them. Therefore, a commonly used representation is needed to be used by people from both fields. MAFR, proposed in this paper, can provide a solution for the communication gap between healthcare professionals and IT developers. The proposed method is applied in speech and language disorder therapy and a mobile application design for the practices were developed. The usefulness and effectiveness of the MAFR were verified through interviews with four therapists. From the results, MAFR happens to be a very useful method that can be applied in healthcare field. It is an effective and efficient model for developing mobile applications in the areas that need special expertise. 


\section{References}

1. Little, A.D.: Capturing Value in the mHealth Oasis: An Opportunity for Mobile Network Operators (2011), http: / / www. adlittle.com/viewpoints.html? \&view=519 (retrieved)

2. Backere, F., Steurbaut, K., Colpaert, K., Decruyenaere, J.: On the Design of a Management Platform for Antibiotic Guidelines in the Intensive Care Unit. In: Fifth International Conference on Software Engineering Advances, Nice, France, August 22-27 (2010)

3. Clancy, H.: The Mobile Health Application Ecosystem: Primary Categories \& Development Consideration. MobileHealthcareToday, Virgo (2011)

4. Dankelman, J., Grimbergen, C.A., Stassen, H.G.: New Technologies Supporting Surgical Interventions and Training of Surgical Skills. IEEE Engineering in Medicine and Biology Magazine, 47-52 (May/June 2007)

5. Dennis, P.C., Baker, L.: Prevalence and type of psychiatric disorderand developmental disorders in three speech and language groups. UCLA Neuropsychiatric Institute, USA (2002)

6. HealthcareITNews, mHealth apps forecast to increase threefold by 2012 (2010), http: / / www. heal thcareitnews. com/news / mhealth-apps-forecast-increase-threefold-2012 (retrieved)

7. Kilov, H., Sack, I.: Mechanisms for communication between business and IT experts. Computer Standards \& Interfaces 31, 98-109 (2009)

8. Ongenae, et al.: Towards computerizing intensive care sedation guidelines: design of a rulebased architecture for automated execution of clinical guidelines. BMC Medical Informatics and Decision Making 10(3) (2010), doi:10.1186/1472-6947-10-3

9. Research2guidance, US\$ 1.3 billion: The market for mHealth applications in 2012 (2012), http: / / www . research2guidance.com/

us-1.3-billion-the-market-for-mhealth-applications-in-2012/ (retrieved) 